\title{
EMPREGO DA AZIDA SÓDICA, COMO CONSERVADOR DE FEZES, PARA A PESQUISA DE OVOS DE SCHISTOSOMA MANSONI PELO MÉTODO DE KATO-KATZ
}

\author{
Elenice Messias do Nascimento Gonçalves, Rubens Campos, \\ Vicente Amato Neto, Pedro Luiz Silva Pinto e Antônio \\ Augusto Baillot Moreira
}

\begin{abstract}
O método de Kato-Katz é muito utilizado para pesquisa de ovos de helmintos nas fezes e em determinadas ocasióes, como por exemplo no trabalho de campo, afigura-se conveniente preservar o material a examinar, com o intuito de facilitar o transporte $e$ operacionalidade.

Na tentativa de poder usar conservador sóliảu, capaz de, em relação a ovos de Schistosoma mansoni, manter a morfologia, impedir a evolução e não interferir no processo de clarificação pela glicerina, os autores utilizaram a azida sódica $\left(\mathrm{NaN}_{3}\right)$, que foi misturada, na quantidade de 2-3mg em aproximadamente $2 \mathrm{~g}$ de fezes de pacientes eliminando número conhecido de ovos, quantificados pelo processo de Kato-Katz. As fezes com preservador ficaram mantidas em temperatura ambiente e foram feitas contagens, pela mesma técnica, apos uma, duas, quatro, oito e doze semanas. As observações, feitas em 53 amostras, demonstraram que em 51 o número de ovos permaneceu, aproximadamente, idêntico e com estruturas conservadas, de molde a permitir o dignóstico. Em dois casos, nas oitava e décima-segunda semanas, as fezes estavam desidratadas, ressecadas e impróprias para a contagem. A azida sódica, portanto, mostrou-se adequada para a conservação de fezes a serem submetidas ao método de Kato-Katz.

Palavras-chaves: Esquistossomose mansônica. Diagnóstico laboratorial. Conservação de fezes. Método de Kato-Katz.
\end{abstract}

$\mathrm{O}$ método de Kato-Kats tem tornado-se uma técnica de apreço, em Parasitologia, por prestar-se à análise qualitativa e quantitativa de fezes, com finalidade de diagnóstico de helmintoses intestinais, demonstrando possuir sensibilidade e reprodutibilidade satisfatórias.

A despeito de ser simples e econômico, encerra a desvantagem de não possibilitar aplicações às amostras fecais preservadas com conservadores tradicionais, os quais promovem liquefação e diluição, prejudicando a execuçāo da técnica. Em matérias fecais não preservadas, mesmo que mantidas refrigeradas e analisadas poucos dias após a coleta, observa-se a alteração do desenvolvimento e degeneração dos ovos de helmintos constituindo isso uma limitação à indicação do método.

Com o objetivo de aperfeiçoamento, modificações foram introduzidas, a fim de contornar tais

\footnotetext{
Laboratório de Investigação Médica-Parasitologia e Divisão de Laboratório Central do Hospital das Clínicas, da Faculdadade de Medicina da Universidade de São Paulo.

Endereço para correspondência: Faculdade de Medicina da Universidade de São Paulo - Laboratório de Investigação Médica-Parasitologia - Avenida Doutor Arnaldo, 4552 . andar - 01246 São Paulo, SP - Brasil.
}

Recebido para publicação em 4/11/87 dificuldades. A adição de conservador adequado, que preserve a morfologia dos ovos, previna a embriogênese, diminua a atividade de microorganismos presentes nas fezes sem alterar a concentração dos ovos e não prejudique o mecanismo de clarificação, mereceu avaliação em estudo anterior levado a efeito por Bundy e cols ${ }^{1}$, que procuraram apreciar méritos e desvantagens da azida sódica quando usada com a finalidade exposta.

Em nosso meio o método de Kato-Katz encontra sua maior aplicação na quantificação de ovos de Schistosoma mansoni presentes nas fezes e essa circunstância levou-nos a avaliar a utilidade do conservador azida sódica no que concerne aos referidos ovos, para facilitar, sobretudo, tarefas diagnósticas e epidemiológicas relacionadas em localidades desprovidas de recursos laboratoriais indispensáveis.

\section{MATERIAL E MÉTODOS}

Compuseram nossa casuística 53 amostras de fezes, comprovadamente com ovos de $S$. mansoni, conforme pesquisas por técnicas rotineiramente empregadas na Divisão de Laboratório Central do Hospital das Clínicas, da Faculdade de Medicina da Universidade de São Paulo. 
Gonçalves EMN, Campos R, Amato Neto V, Pinto PLS, Moreira AAB. Emprego da azida sódica, comoconservadordefezes, para a pesquisa de ovos de Schistosoma mansoni pelo método de Kato-Katz. Revista da Sociedade Brasileira de Medicina Tropical 21: 59-62, Abr-Jun, 1988.

Cada material foi também submetido, individualmente, à análise quantitativa pelo método de Kato-Katz, em sua modalidade clássica ${ }^{3}$. À cada amostra adicionamos, para a comparação, azida sódica $\left(\mathrm{NaN}_{3}\right)$ na proporção de 2 a $3 \mathrm{mg}$ para aproximadamente $2 \mathrm{~g}$, procedemos à mistura das partes e, a seguir colocamos os materiais em frascos de polietileno com tampa, para manutenção em temperatura ambiente $\left(23-26^{\circ} \mathrm{C}\right)$.

Aquilatamos a eficácia do conservante: 1) pela execução do método de Kato-Katz com aliquotas colhidas, respectivamente, em periodos de uma, duas, quatro, oito e doze semanas de armazenamento, sucedendo comparação frente aos valores obtidos na contagem inicial; 2) pela observação de possíveis alterações morfológicas dos ovos e documentação fotográfica dos detalhes interpretados como importantes.

\section{RESULTADOS}

Todas as contagens de ovos, que constituem nossas verificações fundamentais, estão especificadas na Tabela 1.

Tabela 1 - Contagens de ovos de Schistosoma mansoni nas fezes, pelo método de Kato-Katz, sem adição de azida sódica e uma, duas, quatro, oito e doze semanas após o emprego desse conservador.

\begin{tabular}{|c|c|c|c|c|c|c|}
\hline \multirow{2}{*}{$\begin{array}{l}\text { Caso } \\
\text { número }\end{array}$} & \multirow{2}{*}{$\begin{array}{c}\text { Sem } \\
\text { conservador }\end{array}$} & \multicolumn{5}{|c|}{$\begin{array}{c}\text { Após o emprego do conservador } \\
\text { (semanas) }\end{array}$} \\
\hline & & Uma & Duas & Quatro & Oito & Doze \\
\hline 1 & 1176 & 1296 & 1320 & 1176 & 960 & 1152 \\
\hline 2 & 1032 & 936 & 912 & 768 & 768 & 768 \\
\hline 3 & 240 & 216 & 216 & 192 & $*$ & $*$ \\
\hline 4 & 624 & 672 & 552 & 528 & 480 & 552 \\
\hline 5 & 1944 & 2304 & 2064 & 2112 & 2256 & 2184 \\
\hline 6 & 1128 & 1200 & 1320 & 1128 & 936 & $*$ \\
\hline 7 & 1920 & 1680 & 1896 & 2448 & 2400 & $*$ \\
\hline 8 & 696 & 624 & 720 & 648 & 744 & 696 \\
\hline 9 & 192 & 144 & 144 & 144 & 120 & 168 \\
\hline 10 & 1080 & 1344 & 1296 & 1296 & 1368 & 1104 \\
\hline 11 & 888 & 912 & 960 & 960 & 864 & 696 \\
\hline 12 & 744 & 624 & 576 & 552 & 528 & 624 \\
\hline 13 & 523 & 480 & 456 & 408 & 408 & 432 \\
\hline 14 & 216 & 192 & 168 & 168 & 216 & 0 \\
\hline 15 & 528 & 744 & 744 & 672 & 648 & 480 \\
\hline 16 & 1560 & 1440 & 1536 & 1512 & 1392 & 1056 \\
\hline 17 & 1296 & 1128 & 1104 & 1104 & 1248 & 1128 \\
\hline 18 & 360 & 336 & 384 & 360 & 336 & 288 \\
\hline 19 & 456 & 408 & 360 & 360 & 384 & 216 \\
\hline 20 & 2232 & 2376 & 2280 & 2112 & 2184 & 2136 \\
\hline 21 & 624 & 576 & 600 & 552 & 504 & 456 \\
\hline 22 & 1032 & 1248 & 1224 & 1080 & 1032 & 912 \\
\hline 23 & 1296 & 1128 & 1224 & 1104 & 1104 & 1104 \\
\hline 24 & 216 & 192 & 192 & 192 & 0 & 0 \\
\hline 25 & 960 & 840 & 840 & 792 & 648 & 720 \\
\hline 26 & 192 & 192 & 168 & 144 & 168 & 120 \\
\hline 27 & 1992 & 1944 & 1800 & 2040 & 1944 & 2016 \\
\hline 28 & 648 & 696 & 720 & 816 & 816 & 912 \\
\hline 29 & 1872 & 1536 & 1440 & 1416 & 1584 & 1800 \\
\hline 30 & 7456 & 3168 & 4200 & 4704 & 4272 & $*$ \\
\hline 31 & 1248 & 1512 & 1464 & 1464 & 1416 & 1680 \\
\hline 32 & 2688 & 3192 & 2520 & 3024 & 2976 & 3384 \\
\hline 33 & 624 & 504 & 528 & 552 & 504 & 624 \\
\hline 34 & 2664 & 2400 & 2736 & 2760 & 2520 & 2424 \\
\hline 35 & 1584 & 1800 & 1944 & 1608 & 1872 & 1680 \\
\hline
\end{tabular}


Gonçalves EMN, Campos R, Amato Neto V, Pinto PLS, Moreira AAB. Emprego da azida sódica, como conservador de fezes, para a pesquisa de ovos de Schistosoma mansoni pelo método de Kato-Katz. Revista da Sociedade Brasileira de Medicina Tropical 21: 59-62, Abr-Jun, 1988.

Tabela 1 - Continuação.

\begin{tabular}{|c|c|c|c|c|c|c|}
\hline \multirow{2}{*}{$\begin{array}{c}\text { Caso } \\
\text { número }\end{array}$} & \multirow{2}{*}{$\begin{array}{c}\text { Sem } \\
\text { conservador }\end{array}$} & \multicolumn{5}{|c|}{$\begin{array}{c}\text { Após emprego do conservador } \\
\text { (semanas) }\end{array}$} \\
\hline & & Uma & Duas & Quatro & Oito & Doze \\
\hline 36 & 1296 & 1128 & 1584 & 1248 & 1200 & 1272 \\
\hline 37 & 5200 & 5064 & 4704 & 4680 & 3364 & 5400 \\
\hline 38 & 6720 & 7056 & 6528 & 5448 & 6768 & 5400 \\
\hline 39 & 696 & 624 & 576 & 576 & 624 & 384 \\
\hline 40 & 552 & 528 & 504 & 696 & 504 & 528 \\
\hline 41 & 144 & 168 & 192 & 168 & 0 & 0 \\
\hline 42 & 288 & 264 & 312 & 360 & 360 & 336 \\
\hline 43 & 4992 & 4032 & 3160 & 3936 & 2136 & 2376 \\
\hline 44 & 408 & 576 & 600 & 840 & 1104 & 648 \\
\hline 45 & 600 & 576 & 624 & 504 & 528 & 528 \\
\hline 46 & 1152 & 1008 & 1032 & 1008 & 1032 & 1152 \\
\hline 47 & 4344 & 4320 & 4872 & 5064 & 2784 & 3192 \\
\hline 48 & 1920 & 1032 & 1104 & 984 & 1296 & 960 \\
\hline 49 & 3168 & 3168 & 3120 & 2544 & 2904 & 2088 \\
\hline 50 & 1368 & 1320 & 1944 & 1368 & 1224 & 1344 \\
\hline 51 & 1176 & 1104 & 1032 & 1128 & 1152 & 1248 \\
\hline 52 & 528 & 600 & 528 & 672 & 648 & 744 \\
\hline 53 & 7056 & 5952 & 8016 & 8256 & 7632 & 7944 \\
\hline
\end{tabular}

* Material insuficiente.

As fezes conservadas apresentaram-se mais escurecidas, sem produção de gás e com decréscimo considerável do odor, provavelmente em virtude da menor atividade de microorganismos. Observamos, microscopicamente, ovos clarificados (Figura 1) e outros exibindo massa granulosa de cor escura (Figura 2), sendo que tais aspectos não prejudicaram o reconhecimento especifico.

Em duas oportunidades (casos n.' 6 e 7), as matérias fecais estavam ressecadas e desidratadas a partir da oitava semana, exigindo reidratação para a contagem.

A estimativa dos valores numéricos registrados nos diversos periodos estabelecidos não evidenciou diferenças consideráveis, indicativas de cargas parasitárias discrepantes e capazes de gerar incorretas deduções sob os pontos de vista clínico-laboratorial e epidemiológico.

\section{DISCUSSÃO}

As negatividades nas oitava e décima-segunda semanas, respectivamente, em três situações, condizem com as verificações de Knight e cols ${ }^{4} \mathrm{e}$ de Jordan e cols ${ }^{2}$, que admitiram menor sensibilidade em infecções de baixa intensidade; todavia, isso não constituiu uma constante em nossa investigação, pois em três outras ocorrências a pequena quantidade de ovos (menos do que 300 ovos/g de fezes) constatada de início não implicou quedas da sensibilidade nas contagens subseqüentes. É lícito, então, considerar o uso da azida sódica como recurso adequado para conservação das fezes a serem submetidas à técnica de Kato-Katz, valendo a pena lembrar, como detalhe, a ação tóxica da substância, quando ingerida, exigindo essa circunstância cuidado durante os trabalhos com ela efetuados.

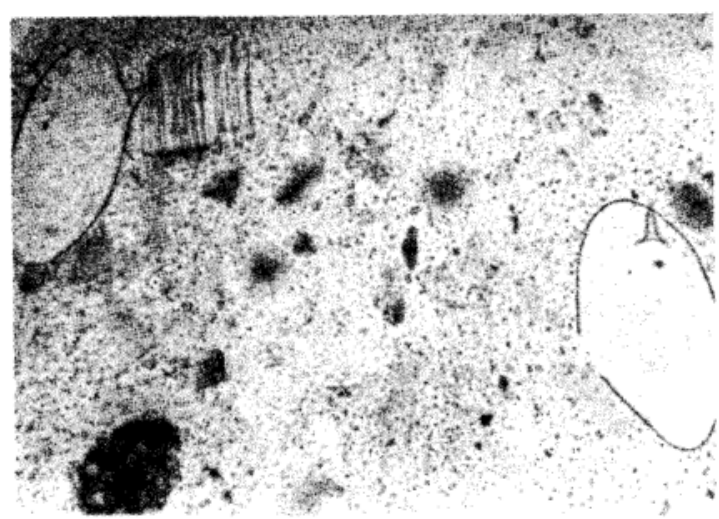

Figura 1-Ovo de Schistosoma mansoni clarificado, 12 semanas após uso da azida sódica para conservação de fezes. 


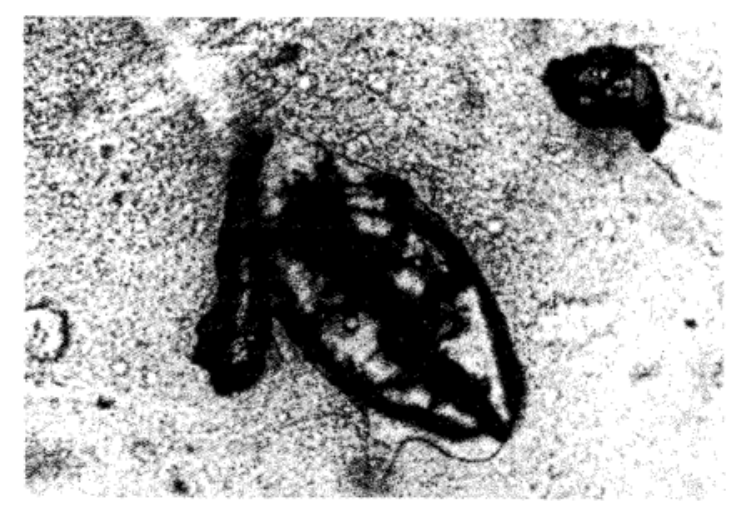

Figura 2-Ovo de Schistosoma mansoni com massa granulosa de cor escura, oito semanas após uso da azida sódica para conservação de fezes.

\section{SUMMARY}

The Kato-Katz technique has been widely employed in the investigation of helminthic eggs in faeces. Sometimes, however, stool specimens must be preserved, specially in field surveys, in order to allow transport and improve feasibility. In an attempt to use a solid preservative, capable of keeping the morphology of Schistosoma mansoni eggs intact, blocking parasite development, without interfering with the usual glycerin clarification method, the authors have added 2-3 $\mathrm{mg}$ sodium azide $\left(\mathrm{NaN}_{3}\right)$ to stools. Each specimen contained about $2 \mathrm{~g}$ stools and was obtained from patients, who eliminated a known egg burden, determined by the Kato-Katz' technique. Stools with sodium azide were kept at room temperature and submitted to subsequent egg counts after one, two, four, eight and twelve weeks. Examinations were carried out in 53 stool specimens and showed that egg structure was preserved and egg counts remained almost identical in 51 of them. Two specimens, examined after eight and twelve weeks, where dehydrated and unsuitable for egg counts. Therefore the authors regard sodium azide as an adequate chemical preservative for stool specimens to be submitted to egg counts by the Kato-Katz' technique.

Key words: Schistosomiasis mansoni. Laboratory diagnosis. Stool preservation. Kato-Katz' technique.

\section{REFERÊNCIAS BIBLIOGRÁFICAS}

1. Bundy DAP, Foreman JDM, Golden MHN. Sodium azide preservation of faecal specimens for $\mathrm{K}$ ato analysis. Parasitology 90:463-469, 1985.

2. Jordan P, Bartholomew RK, Peters PAS. A community study of Schistosoma mansoni egg excretion assessed by the Bell and a modified Kato technique. Annals of Tropical Medicine and Parasitology 75:35-40, 1981.

3. Katz N, Chaves A, Pelegrino J. A simple device for quantitative stool thick-smear technique in schistosomiasis mansoni. Revista do Instituto de Medicina Tropical de São Paulo 14:397-400, 1972.

4. Knight WB, Hiatt RA, Cline BL, Ritchie LS. A modification of the formol-ether concentration technique for increased sensitivity in detection Schistosoma mansoni eggs. American Journal of Tropical Medicine and Hygiene 25:818-823, 1976. 